\title{
ACUERDOS CONJUNTOS: EFECTOS DE SU APLICACIÓN EN COLOMBIA
}

JOINT ARRANGEMENTS: EFFECTS OF MEASURES IN COLOMBIA

\author{
ACORDOS CONJUNTOS: EFEITOS DA APLICAÇÃO NA COLÔMBIA
}

\author{
Por: MELGAREJO MOLINA - Zuray, CIRO ROMERO - Diana Libia
}

Doctorado en Sistemas Flexibles de Dirección de Empresas. Docente Facultad de Ciencias Económicas, Universidad Nacional de Colombia. Email: zamelgarejomo@unal.edu.co - Colombia. Contadora Pública, Universidad Nacional de Colombia. Egresada Facultad de Ciencias Económicas, Universidad Nacional de Colombia. Email: dlciror@unal.edu.co - Colombia

\section{RESUMEN}

Actualmente el país se encuentra en un momento de convergencia hacia las Normas Internacionales de Información Financiera - NIIF, en esa medida este trabajo pretende describir y examinar los negocios conjuntos y las operaciones conjuntas a la luz de tales normas, para ello se llevará a cabo una revisión de los diversos estándares bajo las NIIF Plenas y las NIIF para Pymes tocantes a esta clase de alianzas estratégicas. De dicho estudio se identificarán algunos impactos en la aplicación local de los criterios establecidos para las inversiones en negocios conjuntos bajo los estándares internacionales detectando algunos efectos generales de su aplicación en las empresas colombianas. Por último, se mostrará un caso particular de acuerdo conjunto denominado Joint Venture, vislumbrando los efectos concernientes a la tenencia de este tipo de contrato dentro de una organización.

Palabras clave: Acuerdos conjuntos, Colombia, NIIF, Joint Venture.

JEL: M16, M40, M41, M48. 


\begin{abstract}
Currently the country is in a moment of convergence to International Financial Reporting Standards - IFRS, to that extent this paper aims to describe and discuss joint ventures and joint in the light of such rules operations, for it will conduct a review of the various standards under full IFRS and IFRS for SMEs touching this kind of strategic alliances. In this study, some impacts will be identified in the local application of the criteria for investments in joint ventures under the international standards detecting some general effects of its application in the Colombian companies. Finally, a special case of joint arrangements called Joint Venture is displayed, glimpsing the effects concerning the holding of such contracts within an organization.
\end{abstract}

Keyworks: Joint Arrangements, Colombian, IFRS, Joint Venture.

JEL: M16, M40, M41, M48.

\title{
RESUMO
}

Atualmente o país está em um momento de convergência para as Normas Internacionais de Informação Financeiras - IFRS, nessa medida este trabalho tem como objetivo descrever e analisar os empreendimentos e as operações conjuntas que englobam estas normas; para isso se fará uma revisão dos diversos padrões das NIIF Plenas e as NIFF para PMEs concernentes a este tipo de alianças estratégicas. Neste estudo alguns impactos são identificados na aplicação local dos critérios para investimentos em empreendimentos mistos sob os padrões internacionais, os quais detectam alguns efeitos gerais na sua aplicação em empresas colombianas. Finalmente, um caso particular de acordo conjunto chamado Joint Venture será mostrado, no qual se podem observar os efeitos sobre a realização deste tipo de contrato dentro de uma organização.

Palavras-chave: Acordos conjuntos, Colômbia, NIIF, Joint Venture.

JEL: M16, M40, M41, M48. 


\section{INTRODUCCIÓN}

Desde hace varios años el escenario económico global ha venido transformándose progresiva y rápidamente, esto debido en buena medida al surgimiento de nuevas tecnologías, sistemas de información, procesos, normativas y demás que han permitido a los países estrechar sus lazos comerciales, mediados éstos por pautas internacionales que dan consistencia y legitiman las relaciones económicas mundiales.

No siendo ajeno a este momento de integración comercial y financiera con el resto del mundo, el país afronta actualmente enormes retos estructurales, organizacionales y de estrategia al interior de diferentes sectores nacionales. En particular, Colombia enfrenta hoy la adopción de gran parte de la normativa internacional contable y financiera con el deseo de ser no sólo una nación más competitiva frente a otras sino, además, de alcanzar un nivel de estabilidad económica que traiga consigo un sistema más justo y equitativo para sus habitantes.

La preocupación del Estado por establecer la normativa idónea acorde con las nuevas exigencias globales en materia de regulación, transparencia, información y uniformidad demandada por los diversos grupos de interés que median los mercados, principalmente el de valores, ha llevado en los últimos años a la transformación constante del escenario financiero y contable colombiano; de hecho, la Ley 1314 de 2009 surgió como una de las primeras iniciativas de homogenización de la emisión de información financiera con el propósito de direccionar el proceso de armonización en concordancia con los parámetros establecidos en los estándares internacionales.

Con la promulgación de la mencionada Ley cuyo objetivo es la expedición de normas de contabilidad, de información financiera y de aseguramiento en apoyo a la internacionalización de las relaciones económicas, es decir, que estén en concordancia con los estándares internacionales de reconocida aceptación mundial; surgen diversas nociones desconocidas que han de empezar a ser descritas y estudiadas si se pretende tener éxito en la implementación de las normas internacionales, tal es el caso de los acuerdos conjuntos los cuales concentran la atención del presente escrito.

Así, dado este contexto, cabe esperar que la convergencia hacia los estándares de reconocida aceptación mundial tenga un impacto económico en las organizaciones tocante al tema de la estandarización de sus reportes financieros y, para el caso que atañe a este trabajo, en cuanto a las alianzas estratégicas como fuente de financiación e inversión importante para las empresas a través de las cuales las entidades crean sinergias e incluso sociedades para fortalecer y robustecer su estructura financiera y operativa.

Por lo expuesto, teniendo presente que el concepto de acuerdos conjuntos resulta poco conocido en el país, la aplicación de las normas internacionales en torno a este tema traerá consigo importantes cambios en el actuar de las organizaciones, pues, como afirma López (2014: 31), "las NIIF nos harán ver las cosas de manera diferente [...] tanto internamente, como a nivel de lectores y usuarios de información financiera", por ello, la novedad del concepto en el entorno local y la falta de información relativa al mismo, hace que su estudio seguramente no 
alcance a dimensionar todos los impactos relevantes en su aplicación o incluso es posible que estos resulten ser menos densos de lo esperado.

Pese a las limitaciones enunciadas, este escrito pretende en primera instancia brindar una descripción de algunos factores clave que han de tener en cuenta las organizaciones al implementar las normas internacionales de información financiera (NIIF) junto con ciertos efectos esperados de dicho proceso; posteriormente se realizará una delimitación conceptual de los acuerdos conjuntos a la luz tanto de las NIIF Plenas como de las NIIF para Pymes.

A continuación la atención se centrará en los efectos relativos a la aplicación local de los tipos de acuerdos conjuntos a que hacen referencia las mencionadas normas para, finalmente, caracterizar un tipo de acuerdo conjunto surgido de un contrato bajo la modalidad de Joint Venture el cual será usado, a modo de ejemplo, para describir en un escenario más real el acuerdo conjunto denominado negocio conjunto de tal forma que se puedan vislumbrar algunos de los efectos y alcances de la tenencia de este tipo de inversiones por parte de un ente determinado.

Dado lo precedente, el estudio utiliza el método deductivo ${ }^{1}$ partiendo de un planteamiento general en el cual se describen los tipos de acuerdos conjuntos para enfatizar, posteriormente, en un caso particular de acuerdo reconocido como Joint Venture; para ello, se emplea el estudio de carácter descriptivo de las definiciones, características y demás parámetros concernientes a los acuerdos conjuntos los cuales son detallados ampliamente en los estándares internacionales de información financiera.

\section{ANTECEDENTES}

De la bibliografía encontrada a nivel local se desprende el hecho de que el estudio sobre los acuerdos conjuntos es limitado y se ha basado en la descripción de algunos tipos de operaciones conjuntas ampliamente conocidos como las uniones temporales, los consorcios y algunas clases de alianzas estratégicas. Existen instructivos, manuales e incluso variados artículos que explican algunas de las principales diferencias, impactos, limitaciones y demás que pueden esperarse de la aplicación general de las normas internacionales de información financiera.

Por ejemplo, entidades de carácter público como la Superintendencia de Sociedades y la Superintendencia Financiera, poseen diversos instructivos relativos a la aplicación y descripción de cambios e impactos esperados de la convergencia hacia estándares internacionales. Sin embargo, el tema de los negocios conjuntos ha sido poco explorado y no se encuentran estudios específicos concernientes a esta clase de inversiones ni de los impactos esperados de su aplicación en Colombia.

En contraste, a nivel internacional existen estudios concernientes a los acuerdos conjuntos muy alineados con los propósitos perseguidos en el presente trabajo, en este sentido Arroyo (2012) realiza una descripción del Joint Venture como alianza estratégica enlazando el tema con las pautas de su tratamiento contable según lo

1. La información contenida en este párrafo se basa en definiciones encontradas en las siguientes páginas web: http://metodologia02.blogspot.com/p/operacionalizacion-de-variables.html y http:// www.rena.edu.ve/cuartaEtapa/metodologia/Tema4.html 
establecido en la normativa internacional relativa a los negocios conjuntos. Por su parte, Fernández (2009) realiza una descripción conceptual de los negocios conjuntos ejemplificando sus principales características.

Por otra parte, de la revisión bibliográfica llevada a cabo puede establecerse que son muchos los artículos de carácter nacional e internacional que centran su atención en los beneficios y retos generales implicados en la adopción de los estándares internacionales de información financiera bajo la perspectiva de los autores para sus respectivas naciones, tal es el caso de Rodríguez (2009), Salazar (2013) y Lozada (2015); en el caso particular de las inversiones se ha venido escribiendo sobre el tema de los grupos empresariales y las subordinadas, al respecto puede citarse a Patiño (2007).

Otra de las temáticas tratadas en varios artículos de revista se concentra en las combinaciones de negocios y la consolidación de estados financieros, esto de forma más masiva que respecto al tema de los acuerdos conjuntos; como autores puede nombrarse a De Franchini, Tramallino, Cruz y otros (2008). Pese a lo expuesto, cabe destacar la existencia de algunos escritos en los cuales se describen y pormenorizan ciertos aspectos que atañen al tema de estudio que concentra este trabajo, tal es el caso de los manuscritos mencionados en párrafos precedentes de autores como Arroyo (2012) y Fernández (2009).

\section{FACTORES ESENCIALES EN LA IMPLEMENTACIÓN DE NIIF}

La transición hacia las IFRS como marco de reporte financiero de carácter mundial se ha acelerado más de lo esperado, según una publicación del IFRS Foundation a 2013 casi 120 países han cambiado progresivamente, desde 2001, sus requerimientos locales de información financiera de empresas públicas. Tener un lenguaje global de información financiera genera ventaja a las empresas "que deseen centralizar sus funciones de reporte financiero creando así eficiencias" (2013: 7) al eliminar la necesidad de reportar información financiera consolidada en bases contables múltiples.

La mencionada publicación recalca la posibilidad de reducir costos y fortalecer los controles de reporte al presentar información consolidada de empresas subsidiarias bajo un lenguaje contable uniforme. Además, la adopción de una base común de información financiera entre grupos globales competidores, hace más transparente la comparación de resultados obtenidos generando mayor confianza para los inversionistas y demás sectores interesados.

Así las cosas, siguiendo esta oleada mundial de aceptación y transición hacia un lenguaje homogéneo de reporte de información financiera que, entre otras cuestiones, permite un mayor acceso a los mercados de capitales globales mediante el establecimiento de acuerdos comerciales; el gobierno nacional se ha venido pronunciando al respecto a través de diversas directrices en torno a la implementación de las NIIF mediante la promulgación de nuevas reglamentaciones de aplicación obligatoria las cuales vienen siendo acatadas por parte de los entes nacionales hace ya algún tiempo.

Por lo precedente, si las organizaciones desean realizar dicha transición con el menor traumatismo, han de llevar a cabo un estudio concerniente a los aspectos 
cruciales a tener presente en su implementación de estándares internacionales antes de iniciar su proceso de adopción de dichos requerimientos de tal forma que la incorporación de los parámetros establecidos por las NIIF a su quehacer sea exitosa; dado lo anterior, las empresas han de prestar especial atención a aspectos como los siguientes:

- Identificar las diferencias principales entre las NIIF y los Principios de Contabilidad Generalmente Aceptados (PCGA) locales.

- Seleccionar un equipo capacitado y comprometido con el proyecto de implementación involucrando a las directivas principales de la entidad al igual que al departamento de tecnología encargado de adaptar los sistemas de información a las nuevas necesidades.

- Capacitar en NIIF a todo el personal de la organización.

- Planear una metodología idónea que facilite el proceso de convergencia.

Los aspectos señalados han sido propuestos por Softland Colombia (2013) recomendando, además, la elaboración de un plan estratégico que involucre a todas las áreas afectadas en la compañía pues entre los impactos esperados de la transición a NIIF se prevén, entre otros, la redefinición de políticas contables en las entidades, el manejo de nueva terminología propia de las NIIF Plenas y de las NIIF para Pymes, reducción en el número de estados financieros a presentar de cinco a cuatro, reclasificación de las cuentas de orden, adopción del lenguaje internacional XBRL para el reporte de la información financiera de la compañía, mayor análisis financiero y trabajo en equipo para asegurar el éxito del proyecto (Díaz y Estévez: s.f.).

\section{LOS ACUERDOS CONJUNTOS SEGÚN LA NORMA INTERNACIONAL}

Con la actual política de convergencia hacia normas internacionales de información financiera las entidades colombianas se han visto en la necesidad de replantear la estructura contable y financiera de sus negocios para ser más competitivas y alinear con los cambios reglamentarios que han surgido en época reciente en el país en esta materia, por ello, puesto que el tema central de este escrito atañe a los negocios conjuntos, siendo estos un tipo de acuerdo conjunto conviene revisar las normas internacionales tocantes a este concepto poco estudiado en el ámbito local para, posteriormente, entrar a caracterizar los negocios conjuntos.

\section{NIIF Plenas}

La Norma Internacional de Información Financiera 11 (NIIF, 11) Acuerdos Conjuntos define un acuerdo conjunto como un acuerdo mediante el cual dos o más partes mantienen control conjunto, es decir, las partes están obligadas por un acuerdo contractual que otorga a dos o más de esas partes control conjunto sobre el acuerdo, dicho control es decidido contractualmente y existe solo cuando las decisiones sobre actividades relevantes, aquellas que afectan los rendimientos del acuerdo, precisan del consentimiento unánime de las partes que comparten 
el control lo que implica que ninguna de las partes individualmente posea por sí misma el control del acuerdo (2014: 4, 7).

Ahondando en lo relacionado con el acuerdo contractual, según lo describe la NIIF 11, en este se estipulan las cláusulas que han de cumplir quienes se encuentran sujetos al acuerdo conjunto y en este se tratan temas tales como el propósito, actividad y duración del acuerdo; forma de nombramiento de los miembros del órgano de dirección del acuerdo conjunto; todo lo relacionado con el proceso de toma de decisiones; el capital u otros aportes que se precisen de las partes y la forma en que tales partes comparten los activos, pasivos, ingresos por actividades ordinarias y gastos o resultados del periodo en relación con el acuerdo conjunto establecido (2014: 11).

Tocante al control, la NIIF 10 Estados Financieros Consolidados, determina la existencia de control cuando el inversor reúne los siguientes requisitos (2014: 9):

- Poder sobre la participada

- Derecho a rendimientos variables procedentes de su implicación en la participada

- Capacidad de utilizar su poder sobre la participada para influir en el importe de los rendimientos del inversor

Adicionalmente, la NIIF 10 recalca que dos o más inversores controlan de manera colectiva una participada cuando actúan de forma conjunta al dirigir las actividades relevantes, por tanto, a través de la determinación del control, teniendo presente los requisitos enumerados, es posible establecer si todas las partes o solo un grupo tienen derecho a los rendimientos variables de su participación en el acuerdo y la capacidad de influir en estos mediante su poder sobre el acuerdo.

Debe resaltarse que entre los múltiples propósitos que pueden dar origen a un acuerdo conjunto figuran el acceso a nueva tecnología, mercados o el hecho de que las partes involucradas deseen compartir costos y riesgos (NIIF 11, 2014: 14). Teniendo presente lo anterior, los acuerdos conjuntos contemplados en las NIIF Plenas pueden ser de dos tipos: operaciones conjuntas y negocios conjuntos, la clasificación en alguna de estas clases de acuerdo por parte de una determinada entidad se realiza analizando los derechos y obligaciones de las partes en relación con el acuerdo, además, ante cambios en hechos y circunstancias, la organización debe evaluar nuevamente el tipo de acuerdo en el cual participa.

La NIIF 11 describe un negocio conjunto como "un acuerdo conjunto mediante el cual las partes que tienen control conjunto del acuerdo tienen derecho a los activos netos del acuerdo. Esas partes se denominan participantes en un negocio conjunto" (2014: 7). Adicionalmente, la Norma Internacional de Contabilidad 28 (NIC 28) Inversiones en Asociadas y Negocios Conjuntos especifica que todo negocio conjunto debe contabilizarse utilizando el Método de la Participación Patrimonial (MPP) según el cual debe registrarse la inversión inicial al costo ajustándose posteriormente en razón de los cambios posteriores a la adquisición en la parte poseída por el inversor de los activos netos de la participada (2014: 4). 
En cuanto a las operaciones conjuntas tal norma menciona al respecto que se trata de "un acuerdo mediante el cual las partes que tienen control conjunto del acuerdo tienen derecho a los activos y obligaciones con respecto a los pasivos, relacionados con el acuerdo. Esas partes se denominan operadores conjuntos" (2014: 7). El acuerdo contractual en este caso suele describir la naturaleza de las actividades sujetas al acuerdo y la manera en que los involucrados han de llevarlas a cabo conjuntamente.

\section{NIIF para Pymes}

Las NIIF para Pymes en la Sección 15 Inversiones en Negocios Conjuntos presentan una definición semejante de "negocio conjunto" a la señalada en las NIIF Plenas, en ambas normativas se establece, como característica primordial de un negocio conjunto, el control conjunto entendido como un reparto de control de un acuerdo existente cuando las decisiones relevantes, tanto financieras como operativas, requieren del consentimiento unánime de las partes que comparten el control.

Es de anotar que bajo las NIIF para Pymes los negocios conjuntos abarcan las operaciones controladas de forma conjunta así como las entidades controladas conjuntamente. Respecto a la operación de algunos negocios conjuntos, dicha norma establece que estos implican el uso de activos y otros recursos de los participantes quienes usan su propiedad, planta y equipo y llevan sus propios inventarios incurriendo en gastos y pasivos propios. Además, en las actividades emprendidas de manera conjunta los participantes especifican en el acuerdo del negocio conjunto cómo compartirán los ingresos obtenidos de la actividad ordinaria y cualquier gasto común relativo al acuerdo (2009: 91).

Adicionalmente, se reconoce como negocio conjunto la existencia de una entidad controlada de forma conjunta lo que implica "la creación de una sociedad por acciones, una asociación con fines empresariales u otro tipo de entidad, en la que cada participante adquiere una participación” (2009: 92) la operación de la entidad se da en términos semejantes a la de cualquier otra, salvo por la existencia de un acuerdo contractual en el cual se establece el control conjunto sobre la actividad económica del nuevo ente.

Ahora bien, conviene realizar una revisión de reglamentaciones locales que aunque no hacen referencia a los negocios conjuntos específicamente, presentan conceptos cercanos a dicha noción.

\section{Reglamentación Local}

En la norma local no se encuentra alusión explícita sobre directrices concernientes a los acuerdos conjuntos, específicamente en lo tocante a los negocios conjuntos; sin embargo, se brindan pautas relativas a los consorcios, las uniones temporales, las cuentas en participación, las cuentas de operación conjunta y los patrimonios autónomos como algunas de las acepciones más próximas a este tema. De hecho, en la Circular Externa 6 de 2009 proferida por la Superintendencia de Sociedades, se presenta una descripción sobre los consorcios, las uniones temporales, las cuentas en participación y los contratos de Administración Delegada 
tanto en aspectos técnico-contables como en relación a las revelaciones en notas a estados financieros.

La mencionada circular enuncia, además, que un contrato de colaboración también puede denominarse acuerdo conjunto y brinda una noción de lo que es una operación conjunta en los siguientes términos: "una operación conjunta es un acuerdo que implica el uso de activos y otros recursos de los partícipes, destinados a realizar una actividad comercial o empresarial, como es el caso de fabricar o vender productos" (2009: 4).

Existen otras reglamentaciones como por ejemplo la Circular Externa 5 de 2000 publicada por la Superintendencia de Sociedades donde se brinda orientación respecto a los fundamentos de carácter jurídico y general que han de tener en cuenta las entidades sujetas a consolidar estados financieros los cuales involucran la figura de subordinación que depende, a su vez, del tipo de control ejercido por las entidades controlantes. De igual forma, la Circular Externa 30 de 1997 emitida por la misma superintendencia, ahonda en las obligaciones que recaen sobre aquellas organizaciones que poseen inversiones cuyo porcentaje de participación da lugar a la subordinación actuando la entidad inversionista en calidad de controlante.

De acuerdo a lo precedente, la normativa local no cuenta con nociones semejantes a las planteadas en las NIIF Plenas y en las NIIF para Pymes respecto al tema de los negocios conjuntos, particularmente, de esto se discierne que el país ha de enfrentarse a cambios sustanciales en lo tocante a las inversiones que posea una entidad en razón al tipo de acuerdo conjunto en el que se halle involucrada.

Por lo anterior, es de esperar que los encargados de la convergencia al interior de cada organización se empapen respecto a los requerimientos necesarios para aplicar correctamente las pautas de carácter teórico y técnico en torno a los negocios conjuntos en el escenario local. Igualmente deberán ajustarse las reglamentaciones existentes sobre operaciones y acuerdos conjuntos según se precise para una cabal implementación de los estándares internacionales de información contable y financiera.

\section{PRINCIPALES DIFERENCIAS ENTRE NIIF PLENAS Y NIIF PARA PYMES RESPECTO A LOS NEGOCIOS CONJUNTOS Y LAS OPERACIONES CONJUNTAS}

Las NIIF Plenas presentan una definición semejante de "negocio conjunto" a la señalada en las NIIF para Pymes, en ambas normativas se establece, como característica primordial de un negocio conjunto, el control conjunto entendido como un reparto de control de un acuerdo existente cuando las decisiones relevantes requieren del consentimiento unánime de las partes que comparten el control.

En cuanto al reconocimiento de una inversión por parte de la entidad, las NIIF Plenas establecen dicho reconocimiento desde el momento que el ente determine su participación en un negocio conjunto y para el caso de una operación controlada conjuntamente han de reconocerse los activos, los pasivos, los ingresos de actividades ordinarias y los gastos incurridos en relación con su participación en el acuerdo. Las NIIF para Pymes contemplan un reconocimiento semejante para el caso de las operaciones controladas pero no se pronuncian en relación a los negocios conjuntos. 
Respecto a la medición de una inversión en un negocio conjunto, según lo establecen las NIIF Plenas, debe realizarse utilizando el Método de la Participación. De forma general, las NIIF para Pymes fijan, para aquellas entidades controladas de forma conjunta, la contabilización de participaciones usando modelo del costo, modelo de la participación o modelo del valor razonable estableciendo, a su vez, parámetros distintos en la información que ha de revelar la entidad de acuerdo al modelo escogido al contabilizar sus inversiones.

En términos de revelación, la NIIF 12 Información a Revelar Sobre Participaciones en Otras Entidades exige a la entidad revelar información sobre los juicios y supuestos significativos usados para determinar el control conjunto de un acuerdo; además de la naturaleza y riesgo de sus participaciones junto con los efectos de estas en la situación financiera, los rendimientos financieros y los flujos de efectivo (NIIF 12, 2014).

Por su parte, las NIIF para Pymes además de contemplar requisitos de revelación específicos de acuerdo al método utilizado por la entidad para contabilizar las inversiones controladas de forma conjunta, enfatizan respecto a los negocios conjuntos que debe revelarse la política contable, el importe en libros de la inversión, el valor razonable de dicha inversión y el importe agregado de los compromisos del ente relacionados con el negocio conjunto (Sección 15, 2009).

Ahora bien, en el caso colombiano, como ya se mencionó en párrafos anteriores, no existe referencia explícita a los negocios conjuntos pero sí se alude a conceptos relacionados con este; así por ejemplo, en relación con la contabilización de aportes en negocios de cuentas en participación, estos se registran como inversiones y se exigen registros separados del negocio en cuentas de orden; los aportes en contratos de asociación se registran como anticipos o en otros deudores.

Sumado a lo expuesto, también los activos, pasivos, ingresos y gastos derivados de los contratos de operación conjunta se registran usando criterios similares a los de las inversiones pero no se especifica su contabilización en la normativa existente. La reglamentación colombiana no contempla la aplicación del Método de la Participación Patrimonial para las operaciones conjuntas ni para los negocios de riesgo compartido.

Como puede apreciarse, las diferencias entre la norma local y la internacional en los aspectos de reconocimiento, medición y revelación distan en gran manera, así pues teniendo presente que el país ha decidido converger hacia los estándares internacionales, los cambios que han de aplicarse a los aspectos técnicos que vienen llevándose a cabo en relación con la noción manejada de acuerdo conjunto y de lo que este tipo de contrato implica ha de alinearse con las exigencias contenidas tanto en las NIIF Plenas como en las NIIF para Pymes, luego los cambios no son solo de forma sino también de fondo.

Ahora bien, con la intención de presentar un panorama más amplio de las diferencias establecidas entre la norma local y las normas internacionales de información financiera en relación con las clases de acuerdos conjuntos, la tabla 1 muestra la conceptualización y diferencias mencionadas para el caso de los negocios conjuntos y las operaciones conjuntas. 


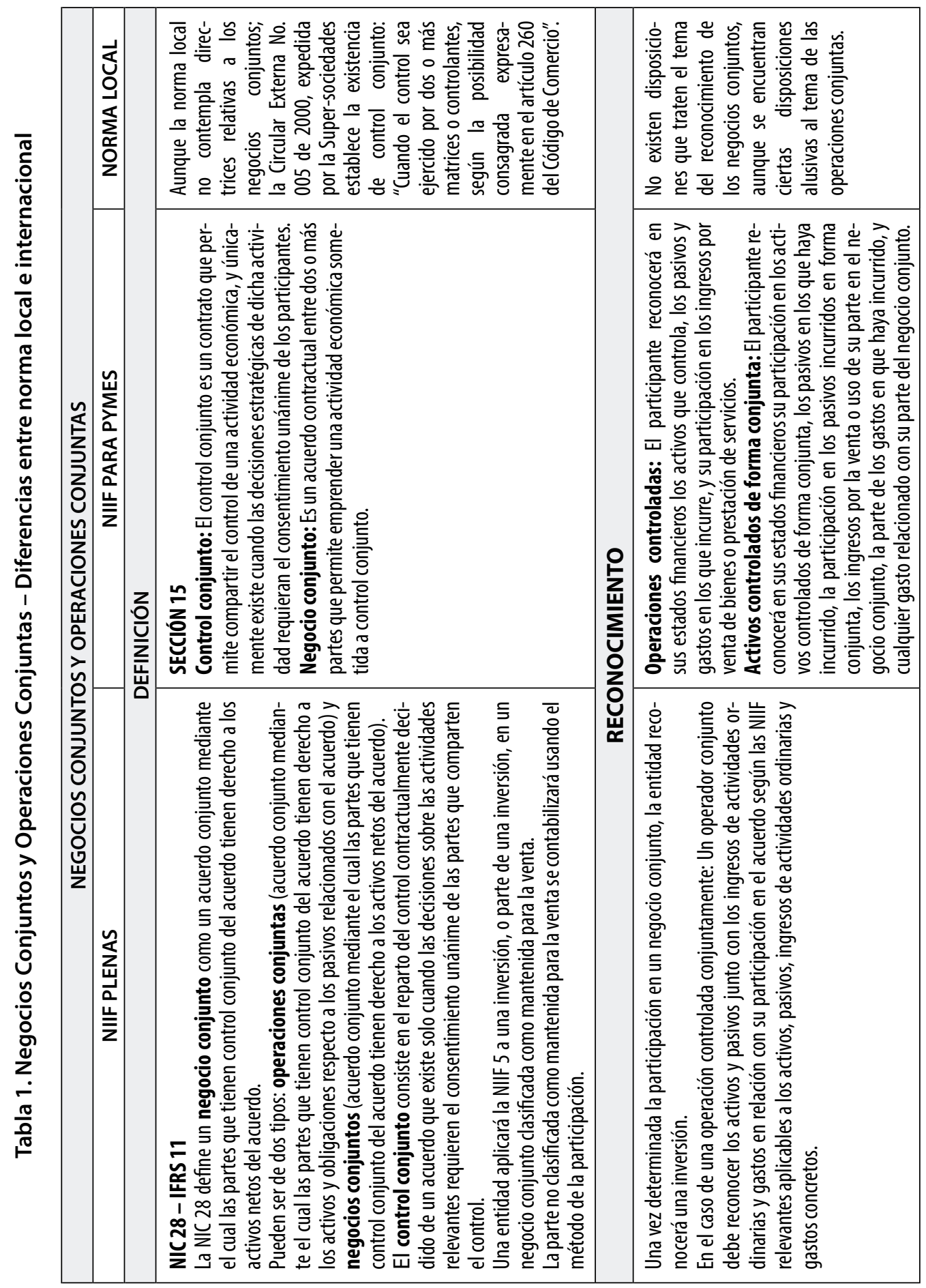




\begin{tabular}{|c|c|c|c|c|c|c|}
\hline 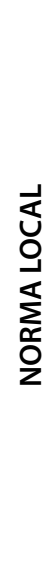 & & 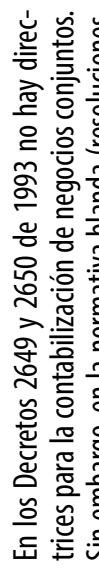 & 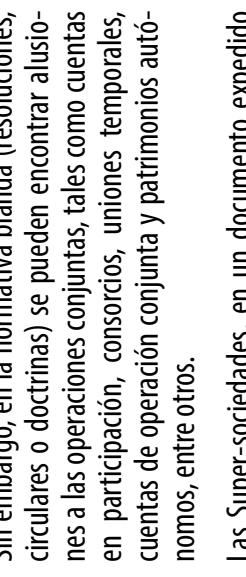 & 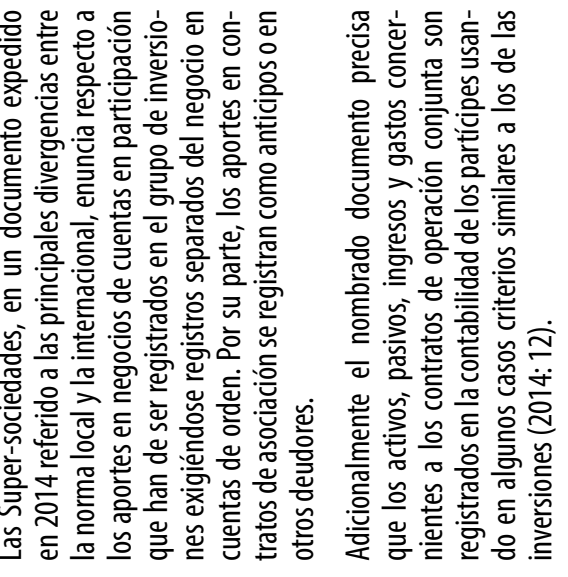 & & 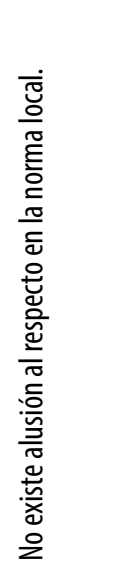 \\
\hline 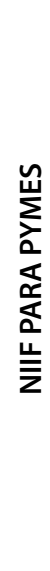 & $\begin{array}{l}z \text { 으 } \\
\text { 믐 } \\
\text { 믈 }\end{array}$ & 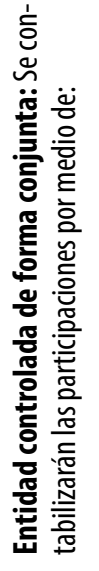 & 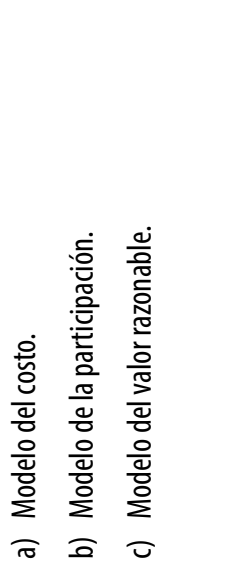 & & 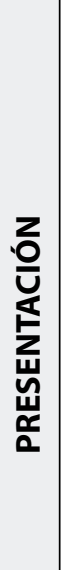 & 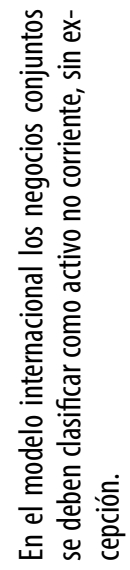 \\
\hline$\overline{\bar{z}}$ & & 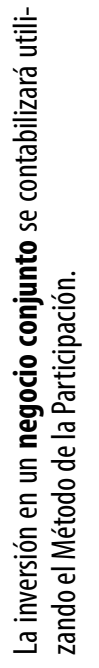 & 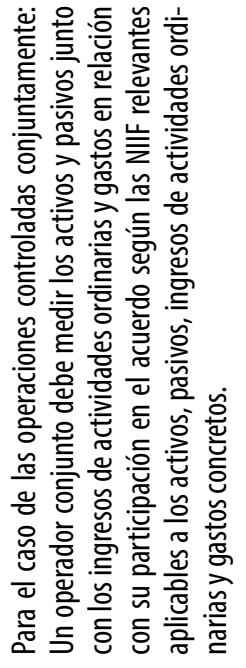 & 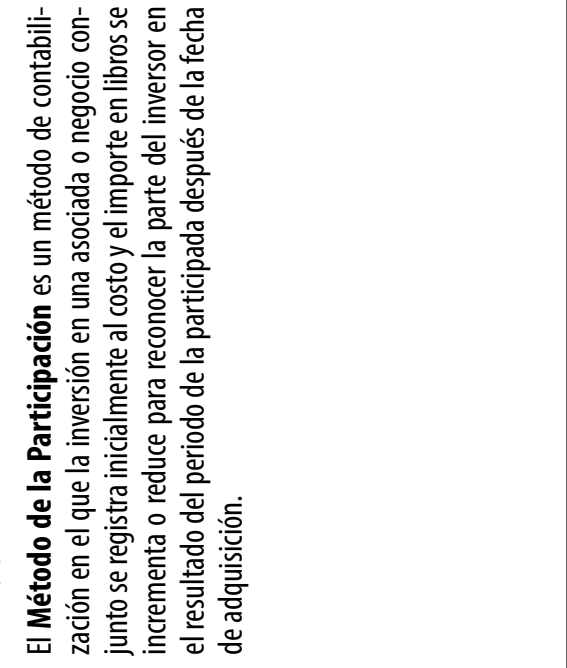 & & 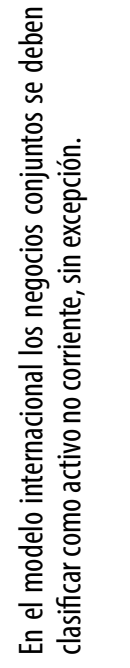 \\
\hline
\end{tabular}




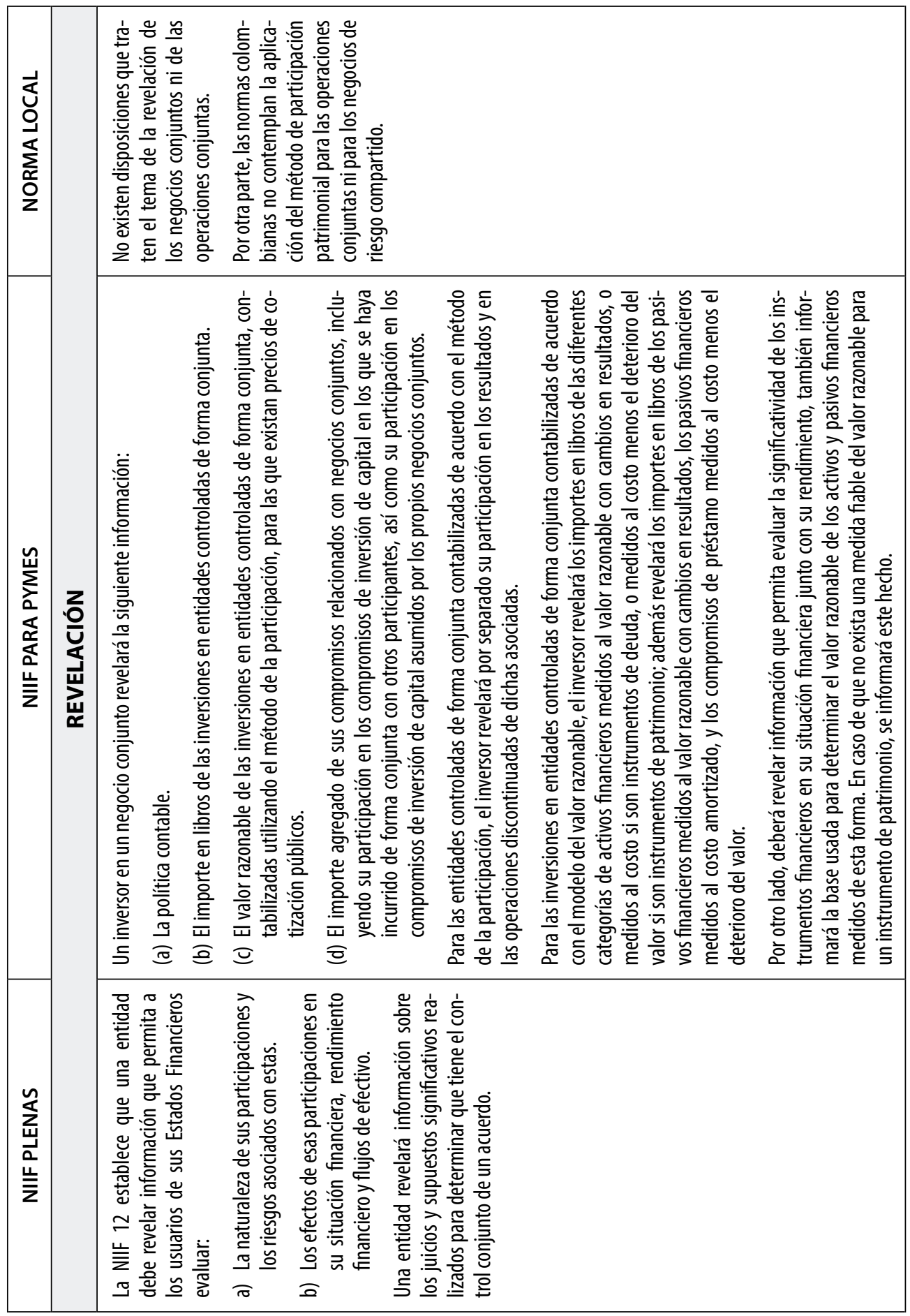




\section{ACUERDOS CONJUNTOS: EFECTOS ESPERADOS POR APLICACIÓN DE LAS NORMAS INTERNACIONALES}

Recordando que la norma local no cuenta con parámetros ni para el reconocimiento de las operaciones conjuntas ni de los negocios conjuntos, salvo ciertas reglamentaciones particulares concernientes a uniones temporales y consorcios, entre otros, donde es permitida la consolidación proporcional, con la aplicación en el contexto local de las normas internacionales de información financiera y contable se prevén ciertos impactos tocantes a los siguientes aspectos:

En el caso de las NIIF Plenas, las operaciones conjuntas y los negocios conjuntos son evaluados a la luz de un contrato donde ha de especificarse tanto el control conjunto y los riesgos compartidos asumidos en razón del contrato entre las partes involucradas. Dicho acuerdo es reconocido por el MPP precisando de menor cantidad de información financiera en contraste con un incremento en la partida de inversiones.

A este respecto, cabe precisar que la diferencia esencial entre las NIIF Plenas y las NIIF para Pymes radica en que estas últimas ofrecen la posibilidad de usar uno de tres métodos para contabilizar las inversiones en entidades controladas de forma conjunta, estos son: el método del valor razonable, el MPP y el método del costo, por ello, el impacto dependerá del modelo escogido por el ente que ha de evaluar previamente el costo-beneficio de cada uno de estos métodos dependiendo la magnitud y las características de la inversión poseída, entre otras variables.

En el caso de las operaciones conjuntas, ha de reconocerse cada partida en proporción a la participación que se tenga por lo que cabe esperar un incremento en las partidas dependiente de la participación poseída. Para los negocios conjuntos, su medición se lleva a cabo por el MPP impactando la inversión y de acuerdo a los cambios experimentados en el patrimonio del negocio conjunto, se pueden prever incrementos ya sea en el costo o en el gasto; también es preciso medir el deterioro luego de los ajustes al MPP.

La presentación de los negocios conjuntos y de las operaciones conjuntas consiste básicamente en realizar la reclasificación de las inversiones en activos no corrientes.

Un punto en el que se presentará gran impacto tiene que ver con las revelaciones exigidas, pues las NIIF tanto Plenas como para Pymes exigen a la entidad la revelación sobre los juicios y supuestos significativos que llevaron a la determinación del control conjunto de un acuerdo, la naturaleza y riesgos asumidos con la participación y los efectos de esta en la situación financiera, los flujos de efectivo y los rendimientos financieros de la entidad. Es pertinente, además, la revelación de información sobre la naturaleza, alcance e impacto financiero de las participaciones poseídas en acuerdos conjuntos y riesgos asociados con este tipo de contratos.

En el tema de revelaciones las NIIF para Pymes contemplan revelaciones más detalladas dependiendo del método escogido por la organización para conta- 
bilizar su inversión en negocios conjuntos debiendo revelar incluso la política contable, el importe en libros de la inversión, su valor razonable y el importe agregado de los compromisos del ente en relación con el negocio conjunto en el cual participa.

\section{JOINT VENTURE COMO ALIANZA ESTRATÉGICA Y ACUERDO CONJUNTO}

Centrando ahora la atención en los contratos de Joint Venture, es preciso realizar antes algunas precisiones en torno a las alianzas estratégicas, más aún teniendo en cuenta que hoy por hoy estas alianzas se han convertido en potente herramienta para las organizaciones al posibilitar el desarrollo empresarial, la mejora de la competitividad, la creación de nuevos productos y servicios, la exposición a nuevos mercados, la reducción en costos y la opción de compartir riesgos de la mano con otras entidades. Como fenómeno económico, se considera una forma diferente de hacer negocios generando mayores rendimientos con el agregado de generar aprendizajes importantes según el socio elegido.

De forma general, una alianza puede entenderse como un acuerdo entre dos o más organizaciones que sin fusionarse crean una interrelación para aumentar sus ventajas competitivas, desarrollando esfuerzos conjuntos de colaboración para el alcance de objetivos a corto y largo plazo (Molina y Vázquez, 2008). Las alianzas suelen establecerse exclusivamente para llevar a cabo una actividad empresarial específica, por tanto, son temporales.

Las organizaciones emprenden alianzas con miras a alcanzar diversos objetivos estratégicos, Molina y Vázquez (2008) aluden a los siguientes: como medio para facilitar la entrada a un mercado extranjero, para compartir costos fijos y riesgos asociados presentes en el desarrollo de nuevos productos o procesos, como oportunidad de agrupar habilidades y activos complementarios que las empresas no podrían desarrollar de forma independiente y como ayuda para que las entidades establezcan estándares tecnológicos para su industria redundando estos en beneficio propio para el ente.

Asimismo, Arroyo (2012) describe algunas de las razones para emprender alianzas estratégicas, a saber:

Aprovechar el principio de sinergia según el cual el "resultado producido por dos partes trabajando en conjunto es mayor al que hubieren dado cada una por separado" (p. 4), luego al buscar socios estratégicos las entidades aprovechan las capacidades, experiencia y habilidades poseídas por cada organización.

Distribuir los costos y riesgos, así, ante pérdidas o imprevistos que puedan ocurrir, es menos probable el colapso de la entidad, la situación se hace llevadera cuando la obligación es compartida.

Crear economías de escala al ahorrar en recursos incrementando la rentabilidad de los participantes involucrados en el acuerdo. 
Comercializar productos creando canales de distribución eficiente, incluso en lugares geográficos de difícil acceso para empresas individuales.

Ganar posiciones en el mercado al entablar alianzas estratégicas con socios clave, experimentados o que posean influencia en un área específica nueva.

Enfrentar a la competencia especialmente cuando existen empresas fuertemente posicionadas en el mercado.

Aunque existen diversas clases de alianzas estratégicas, este trabajo centra su atención en uno de los tipos de alianza estratégica más común: el Joint Venture -Empresa conjunta- que, según lo describen Molina y Vázquez (2008), consiste en una entidad propiedad de dos partes que cuentan cada una con una participación accionaria del 50\% compartiendo el control de las operaciones entre ambas empresas.

Por su parte, Chavarría define esta clase de contrato de la siguiente forma:

El Joint Venture es aquella asociación temporal entre dos o más personas físicas o jurídicas con miras a la participación en un negocio conjunto constituido por un proyecto específico previamente determinado, para el que ambos realizan aportes de diversa índole y además los mismos se involucran directamente en su administración conjunta (1990: 29-30).

Adicionalmente, para Arroyo (2012), el Joint Venture constituye un contrato mercantil moderno al que le son aplicables las pautas establecidas para los negocios conjuntos en términos contables, estas alianzas poseen un ánimo de lucro, luego los participantes tienen derecho a percibir utilidades, además, el negocio es controlado de forma conjunta y el Joint Venture es individualizable de las entidades que le dieron origen, se distingue de los socios que lo crearon.

En cuanto a los contratos de Joint Venture, estos poseen diversas características entre las cuales se pueden destacar: naturaleza contractual, no poseen naturaleza societaria, contrato atípico surgido de la necesidad de las entidades de realizar alianzas comerciales que les permitan crecer en un mercado competitivo, limitación temporal, beneficios para las partes manifiestas en el valor agregado surgido de su integración al establecer un acuerdo justo donde cada socio reciba beneficios proporcionales a su nivel de inversión durante el transcurso de la alianza, administración del negocio por parte de los participantes en el mismo (Arroyo, 2012).

Este tipo de contrato posibilita la creación de negocios para los cuales no se tienen los recursos necesarios, por ello, quien posee la idea busca socios que puedan soportar materialmente el negocio estando dispuestos a compartir los riesgos e inversión necesaria. Para evitar problemas, las cláusulas establecidas en el contrato deben ser claras, específicas y de acuerdo mutuo entre las partes involucradas. Algunos estudios han arrojado que el éxito en este tipo de alianzas depende en gran medida del conocimiento de las necesidades de los socios participantes a lo largo del tiempo, lo que brinda estabilidad al negocio, además de aspectos como 
el compromiso, la confianza, el reconocimiento de la interdependencia y la comunicación entre socios.

Ahora bien, el Joint Venture como alianza estratégica de la cual surgen transacciones económicas está ceñido a los principios y normas contables que le sean aplicables, a este respecto, la NIC 28 Inversiones en Asociadas y Negocios Conjuntos, contempla que todas las entidades que sean inversoras con control conjunto han de aplicar esta norma, pues dicho control es definido como el reparto del control contractualmente decidido de un acuerdo existente cuando las decisiones sobre actividades relevantes precisan del consentimiento unánime de las partes que comparten el control (2014: 4), tal es el caso en los contratos de Joint Venture. A su vez, la NIIF 11 Acuerdos Conjuntos, define un negocio conjunto como un acuerdo conjunto en el cual las partes que poseen el control conjunto del acuerdo (los partícipes) tienen derecho a los activos netos del acuerdo (2014).

De lo anterior se deduce que siendo el Joint Venture un tipo de alianza estratégica en la cual los socios de un determinado negocio ejercen control sobre la entidad de forma conjunta, las normas especificadas en el párrafo precedente le son completamente aplicables puesto que parten de elementos como el control conjunto y los acuerdos contractuales. Sumado a lo anterior, dado que existen diversas formas y estructuras al realizar un contrato de Joint Venture según los objetivos perseguidos, las normas internacionales reconocen dos tipos de acuerdos conjuntos, a saber: las operaciones conjuntas y los negocios conjuntos.

A continuación se especificará, para cada uno de estos casos, algunos aspectos a nivel contable que han de tenerse en cuenta según la modalidad de contrato escogido.

\section{Contrato para llevar a cabo una actividad conjunta - Operación Conjunta}

En esta clase de acuerdo conjunto los operadores conjuntos utilizan sus activos y recursos propios para el alcance de los objetivos propuestos sin que surja una nueva entidad jurídica; un ejemplo clásico de operación conjunta descrito por Mesén (2008), se presenta cuando un productor de bebidas gaseosas realiza un acuerdo con un distribuidor de productos de consumo masivo para que distribuya en el territorio nacional las bebidas producidas por el primero; así, aunque se ha suscrito una alianza estratégica, cada uno de los empresarios maneja de manera independiente sus activos y pasivos junto con el registro, también independiente, de los ingresos, costos y gastos incurridos en razón del acuerdo conjunto realizado entre los participantes.

Según lo descrito, cada uno de los participantes en el acuerdo administra de manera independiente sus activos, pasivos, ingresos, costos y gastos reconociendo en sus estados financieros, según lo determina la IFRS 11 Acuerdos Conjuntos (2014), sus activos y pasivos junto con su participación en los activos mantenidos y los pasivos incurridos conjuntamente; sus ingresos de actividades ordinarias 
obtenidas de la venta del producto que realiza la operación conjunta junto con los gastos, incluyendo su participación en los gastos incurridos conjuntamente.

Puesto que cada entidad involucrada en la operación conjunta realiza sus propios estados financieros, no es necesario hacer ajustes ni consolidar la información financiera de las empresas incluidas en el acuerdo conjunto pues los efectos de la participación en la alianza suscrita quedan a nivel de los estados financieros individuales. Sin embargo, Arroyo (2012) opina que puede ser conveniente elaborar informes contables para la dirección permitiendo así evaluar el rendimiento del negocio llevado de forma conjunta.

\section{Contrato que da origen a una entidad jurídica nueva - Negocio Conjunto}

Un acuerdo contractual bajo la modalidad de Joint Venture puede dar origen a un ente autónomo independiente de las empresas suscriptoras de la alianza las cuales deciden constituir dicha entidad para administrarla de forma conjunta, teniendo los partícipes del acuerdo el control conjunto sobre la actividad económica que desarrollará la sociedad creada. Aún así, el ente constituido al poseer una estructura financiera independiente tendrá su propio sistema contable y deberá presentar estados financieros individuales acorde con las reglamentaciones contables que rijan en el lugar donde lleve a cabo su actividad económica.

Asimismo, al ser la entidad un ente jurídico sujeto a derechos y obligaciones, podrá incurrir en negociaciones, suscribir contratos, obtener financiamiento para proyectos y realizar todas aquellas actividades avaladas por ley para cumplir con su objeto comercial.

Adicionalmente, en lo relativo al registro contable de los negocios conjuntos, la IFRS 11 Acuerdos Conjuntos especifica lo siguiente:

Un participante en un negocio conjunto reconocerá su participación en un negocio conjunto como una inversión y contabilizará esa inversión utilizando el método de la participación de acuerdo con la NIC 28 Inversiones en Asociadas y Negocios Conjuntos a menos que la entidad esté exenta de aplicar el método de la participación tal como se especifica en esa norma (2014: 8-9).

Por lo expuesto, existe la obligación de utilizar el método de la participación al contabilizar un negocio conjunto bajo NIIF Plenas; dicho método consiste, según lo precisa la NIC 28 Inversión en Asociadas y Negocios Conjuntos, en el registro de la inversión inicial al costo ajustando esta posteriormente según los cambios experimentados tras la adquisición en la parte del inversor de los activos netos de la participada, es decir, posterior al registro inicial al costo, periodo a periodo deben reconocerse los incrementos o decrementos en la porción que corresponde al inversor de los resultados de la participada desde la fecha de adquisición de la respectiva inversión en el negocio conjunto. Sumado a lo anterior, el inversor 
debe reconocer en su resultado la parte que corresponda a las ganancias o pérdidas arrojadas por el negocio conjunto (Arroyo, 2012).

Por otra parte, es importante destacar, en contraste con las exigencias de las NIIF Plenas, el hecho de que las NIIF para Pymes permiten escoger uno de entre tres métodos de contabilización para medir las inversiones en entidades controladas de forma conjunta: el método de la participación, el método del costo y el método del valor razonable; dependiendo de la escogencia realizada por la entidad surgirán diversos impactos, por ello, esta deberá hacer una evaluación del costo-beneficio de cada uno de estos métodos dada la magnitud de la inversión poseída.

Es relevante precisar respecto a cada modelo los siguientes aspectos:

La aplicación del método a valor razonable reviste un costo excesivo, además de mayor complejidad al valorar las inversiones poseídas, en especial cuando las entidades no cotizan en bolsa.

En cuanto a la aplicación del modelo del costo, éste se aplica cuando no existe control; localmente se presenta cuando la participación es menor al 50\%, pero en norma internacional se presume la existencia de control con una participación hasta del $20 \%$ con la existencia de influencia significativa, figura no reconocida en Colombia. Además, localmente se realizan valorizaciones al aplicar el Método del Costo, dicha partida no es utilizada en las NIIF donde se ajusta directamente el activo, sin partidas intermedias.

En caso de utilizarse el método de participación patrimonial existirá una diferencia en cuanto a la situación en la cual se aplica, puesto que en la normativa colombiana se usa cuando existe control asociado al porcentaje de participación; en tanto en la normativa internacional es de aplicación en inversiones en asociadas, así como en operaciones conjuntas, sin que el porcentaje de participación sea el único parámetro a tener en cuenta sobre la existencia de situaciones que pueden incidir en el poder del inversionista sobre la participada (Patiño, 2007).

Así las cosas, el impacto en la entidad dependerá de la política de reconocimiento adoptada, luego la corporación deberá evaluar la magnitud de su inversión, la información que precisa y los costos generados por el método elegido.

\section{CONCLUSIONES}

Como se ha intentado vislumbrar a través de este escrito, el tema de los negocios conjuntos genera expectativa y curiosidad en el ámbito local dada su relevancia en términos estratégicos para las empresas que los poseen, además constituyen una figura nueva que ha de estudiarse e interpretarse antes de ser utilizada por los entes económicos para así generar, a partir de estos acuerdos conjuntos, alianzas que verdaderamente beneficien a sus participantes al fortalecer sus negocios mediante la creación de posibilidades de expansión y aprovechamiento de oportunidades valiéndose de políticas contables y financieras acordes a los cambios normativos que vienen dándose en Colombia en los últimos años. Luego, cabe esperar que los 
impactos por aplicación de las NIIF resulten en cambios palpables y contundentes sobre los estados financieros presentados por las entidades, especialmente sobre las partidas relativas a Inversiones.

Por lo expuesto, en concordancia con las normas internacionales de información contable y financiera que están siendo implementadas en el país, las entidades poseedoras de negocios conjuntos deberán revelar su política contable, el importe en libros de las inversiones, el importe agregado de sus compromisos, los juicios y supuestos por los cuales se presume la existencia de control conjunto junto con aspectos relacionados con la naturaleza, los riesgos de las participaciones, los efectos en la situación financiera, en los rendimientos y en los flujos de efectivo de la entidad.

Adicionalmente, las organizaciones deben presentar aquella información específica exigida en concordancia con el método escogido para contabilizar sus inversiones, a saber: el método del costo, el método del valor razonable o el método de la participación patrimonial. Así las cosas, las empresas deberán analizar los pros y contras de cada uno de los tres métodos referenciados, escogiendo el que mejor se adapte al tipo de inversión y a las necesidades de la entidad según su balance realizado de costo-beneficio y con base en los diferentes impactos que puede generar el método elegido, por ejemplo, al usar el método de la participación patrimonial cobra vital importancia la revelación de información confiable, además deben cumplirse ciertas características, principalmente el uso de las mismas políticas y la depuración de transacciones entre las entidades que intervengan en el negocio conjunto.

Por su parte, al escogerse el modelo del valor razonable ha de evaluarse previamente el costo-beneficio de su aplicación, dada la complejidad que representa tal valoración y los costos excesivos que esto pueda implicar. Entre tanto, de aplicarse el método del costo, la entidad ha de tener presente los parámetros establecidos en la normativa internacional en lo referente a los porcentajes de participación e influencia significativa, pues desde el 20\% de participación se ejerce influencia en una entidad, según lo establecen tanto las NIIF Plenas como las NIIF para Pymes; este hecho es nuevo en Colombia donde se habla de influencia a partir de una participación igual o superior al 50\%.

De forma general, es preciso recordar que la información a revelar tanto en NIIF Plenas como en NIIF para Pymes será más amplia, y tendrá como objetivo permitir a los usuarios de los estados financieros evaluar la naturaleza, alcance, efectos y riesgos derivados de sus inversiones en negocios conjuntos, particularmente.

Por otra parte, en cuanto a las alianzas estratégicas, cabe resaltar que desde hace ya algunos años el mundo viene sufriendo una acelerada globalización la cual ha afectado la economía actual, particularmente se observa el surgimiento de alianzas organizacionales las cuales son posibles por su funcionamiento en redes para competir en momentos específicos; la empresa ya no es hoy la unidad económica sino lo es el proyecto empresarial. Las compañías deben estar en capacidad de 
adaptarse al cliente y sus necesidades, deben tener una producción flexible acorde a lo que demande el mercado (Castell, 2000).

Puesto que los empresarios se ven obligados a mantener el interés de sus clientes, a satisfacer su demanda antes que la competencia conservando la calidad y los precios bajos funcionando bajo los nuevos enfoques de gestión que persiguen, entre otras, la expansión en el mercado; las entidades han visto en la creación de alianzas estratégicas como el Joint Venture, la posibilidad de subsistencia a largo plazo, la generación de un mayor índice de productividad y la oportunidad de alcanzar objetivos que no lograrían de forma individual.

Por lo expuesto, como alianza estratégica de la cual surgen transacciones económicas importantes para las empresas que participan del acuerdo conjunto bajo la modalidad de Joint Venture, esta clase de acuerdo contractual resulta ser una variable decisiva para aumentar el nivel de competitividad de un ente al momento de introducir productos en un mercado nuevo reduciendo costos, aumentando la velocidad de reacción junto con la eficiencia y promoviendo la innovación en la producción de bienes y servicios con un alto valor agregado.

La creación de este tipo de sinergia genera una transición adecuada hacia las exigencias del escenario global pues no basta con dejarse globalizar sino que es prioritario saber hacerlo, de ahí que puedan verse reflejados en el Joint Venture algunos de los parámetros normativos a nivel contable que rigen actualmente en el ámbito internacional, abarcando principios y normas contables que siendo bien interpretados y aplicados garantizarán el éxito del negocio conjunto en el cual participe una determinada entidad.

Reste decir, como aporte, que este documento pretende contribuir a la literatura $\mathrm{y}$ a posteriores investigaciones en torno al tema de los negocios conjuntos, ofreciendo un resumen sobre los principales parámetros establecidos en las normas internacionales de información financiera y contable respecto a la figura conocida como negocio conjunto, brindando a la vez un análisis de algunos de los posibles impactos generados de la aplicación por primera vez en Colombia de este tipo de acuerdo conjunto el cual es desconocido en el escenario local.

Puesto que no existe, hasta el momento, reglamentación local explícita tocante al manejo contable de los negocios conjuntos, se deduce la importancia de este escrito teniendo en cuenta que este nuevo concepto ha de ser interpretado y aplicado en el país por aquellas entidades que posean negocios conjuntos pues deberán poner en práctica los parámetros definidos tanto en las NIIF Plenas como en las NIIF para Pymes para esta clase de inversiones. Adicionalmente, este estudio abre las puertas al debate académico tocante a las ventajas o desventajas de la implementación de los parámetros establecidos por la norma internacional en cuanto al tema de los negocios conjuntos en el escenario local. 


\section{REFERENCIAS}

1. ARROYOC., Yennifer (2012). Alianzas de cooperación estratégica: la figura del Joint Venture y su perspectiva contable-financiera según la“NIC 31: participación en negocios conjuntos". En: Revista Posgrado y Sociedad, Vol. 12, Núm. 2, pp. 1-19. Disponible en: http://web.uned.ac.cr/revistas/ index.php/rps/article/view/106/pdf.

2. CASTELL, M. (2000). Globalización, sociedad y política en la era de la información. En: Bitácora, pp.42-53. Disponible en: file:///C:/Documents\%20 and\%20Settings/Administrador/Mis\%20documentos/Downloads/Dialnet-GlobalizacionSociedadYPoliticaEnLaEraDeLalnformaci-4008342.pdf

3. CHAVARRÍA, J. (1990). Las empresas de participación conjunta o Joint Venture. Tesis para optar por el grado de Licenciado en Derecho. Costa Rica, Facultad de Derecho, Universidad de Costa Rica.

4. CIOAL.COM (2013). Softlan Colombia enumeró los factores clave para adoptar con éxito las NIIF en las empresas. En: Boletín Instituto Nacional de Contadores Públicos de Colombia (INCP), Núm. 93, p. 7.

5. CONGRESO DE LA REPÚBLICA (13 de Julio de 2009). Ley 1314. Bogotá D.C., Colombia. Disponible en: http://actualicese.com/normatividad/2009/ Leyes/L1314-09.pdf.

6. DE FRANCHINI, B., TRAMALLINO de GUINEA, M., CRUZ DE INNOCENTE, CARBAJAL, M. y DE CASTRO, I. (2008). El universo de las combinaciones de negocios - consorcios de cooperación. En: Contabilidad, Vol. 14, Núm. 30-31, pp. 7-24. Disponible en: http://nulan.mdp.edu.ar/1276/1/ FACES_n30-31_7-24.pdf.

7. DÍAZ HENAO, J. y ESTÉVEZ, Y. (S.F.). Normas Internacionales de Información Financiera (NIIF) y su impacto en las Pymes. Disponible en: http:// www.bancoldex.com/documentos/4734_NIIF_presentaci\%C3\%B3n_Bancoldex.pdf.

8. FERNÁNDEZ FERNÁNDEZ, J. (2009). Las inversiones en negocios conjuntos. Pecvnia, p. 401-425. Disponible en: file:///C:/Documents\%20and\%20 Settings/Administrador/Mis\%20documentos/Downloads/Documat-LasInversionesEnNegociosConjuntos-3007718.pdf.

9. INTERNATIONAL ACCOUNTING STANDARD BOARD [IASB]. (2014). Norma Internacional de Contabilidad [NIC] 28: Inversiones en Asociadas y Negocios Conjuntos. Disponible en: http://www.aplicaciones-mcit.gov.co/niif/niif.php?anio_id=2012.

10. INTERNATIONAL ACCOUNTING STANDARD BOARD [IASB] (2014). Norma Internacional de Información Financiera [NIIF] 10: Estados Financieros Consolidados. Disponible en: http://www.aplicaciones-mcit.gov.co/niif/niif.php?anio_id=2012.

11. INTERNATIONAL ACCOUNTING STANDARD BOARD [IASB] (2014). Norma Internacional de Información Financiera [NIIF] 11: Acuerdos Conjuntos. Disponible en: http://www.aplicaciones-mcit.gov.co/niif/niif.php?anio_id=2014.

12. INTERNATIONAL ACCOUNTING STANDARD BOARD [IASB] (2014). Norma Internacional de Información Financiera [NIIF] 12: Información a Revelar Sobre Participaciones en 0tras Entidades. Disponible en: http://www.aplicaciones-mcit.gov.co/niif/niif.php?anio_id=2014.

13. INTERNATIONAL ACCOUNTING STANDARD BOARD [IASB] (2009). Sección 15 - Inversiones en Negocios Conjuntos. En: Normas Internacionales de Información Financiera [NIIF] para Pequeñas y Medianas Entidades [PYMES]. London, United Kindgdon: IASC Foundation Publications Departament. Disponible en: http://www.aplicaciones-mcit.gov.co/niif/niif.php?anio_id=2012.

14. LÓPEZ, A. L. (2014). Contabilidad financiera. Prácticas en los proyectos de implementación de las Normas de Información Financiera (NIF). En: El Contador Público. Instituto Nacional de Contadores Públicos de Colombia (INCP), Núm. 172, pp. 30-38.

15. LOZADA RIVERA, A. (2015). Ruta seductora hacia la convergencia divulgación: NIIF para Pymes. En: Revista Internacional Administración \& Finanzas, Vol. 8, Núm. 2, pp. 83-103. Disponible en: file:///C:/Users/alba/Downloads/SSRN-id2500525.pdf.

16. MESÉN FIGUEROA, V. (2008). Alianzas estratégicas: Su naturaleza, características y criterios de contabilidad. En: TEC Empresarial, Vol. 2, Núm. 1, pp. 29-33. Disponible en: http://tecdigital.tec.ac.cr/servicios/ojs/index.php/tec_empresarial/article/view/772/694.

17. MÉTODOS DE LA INVESTIGACIÓN (2010). Disponible en: http://metodologia02.blogspot.com/p/metodos-de-la-inventigacion.html.

18. MOLINA MARTíNEZ, R. y VÁZQUEZ OLARRA, G. (2008). Los elementos de éxito en la formación de alianzas estratégicas. En: INCEPTUM, Vol. 5, Núm. 5, pp. 15-26. Disponible en: http://www.inceptum.umich.mx/index.php/inceptum/article/view/44/42. 
19. MORÁN SÁMANO, A. (2013). Implementación de las IFRS: Estatus y futuro. Boletín Instituto Nacional de Contadores Públicos de Colombia (INCP). En: Revista Contaduríapublica.org.mx, Núm. 94, pp. 6-8. Disponible en: http://contaduriapublica.org.mx/wp-content/uploads/moran.pdf.

20. PATIÑO, R. A. (2007). Grupos empresariales y la representación de sus inversiones en acciones: comparación entre los IAS - IFRS y la normativa colombiana. En: Revista Activos, Núm. 12, pp. 25-44. Disponible en: http://contaduria.usta.edu.co/images/documentos/activos/12-2.pdf.

21. RODRÍGUEZ LÓPEZ, A. (Abril de 2009). Impacto del nuevo PGC sobre el activo no corriente de las entidades no lucrativas. En: Partida Doble, Núm. 209, pp.40-49. Disponible en: http://nicniif.org/files/u57/ARTICUL0_PARA_DESCARGAR_PDF_ABR_09_4.pdf.

22. SALAZAR BAQUERO, E. (2013). Efectos de la implementación de las NIIF para las PYMES en una mediana empresa ubicada en la ciudad de Bogotá. En: Cuadernos de Contabilidad, Vol. 14, Núm. 35, pp. 395-414. Disponible en: http://www.scielo.org.co/pdf/cuco/v14n35/v14n35a03.pdf.

23. SUPERINTENDENCIA DE SOCIEDADES (2014). Anexo 2. Principales Divergencias en Políticas Contables entre los PGCA en Colombia y las IFRS - NIIF. Disponible en: https://www.supersociedades.gov.co/asuntos-economicos-y-contables/procesos-de-convergencia-niifs/estadistica-de-laconvergencia/Documents/Anexo_2_Principales_Divergencias_En_Politicas_Contables_Entre_PGCA-Col_y_Las_IFRS_NIIF.pdf.

24. SUPERINTENDENCIA DE SOCIEDADES (23 de diciembre de 2009). Circular Externa No. 006. Contratos de Colaboración. Disponible en: http://www. supersociedades.gov.co/imagenes/Circular\%20Externa\%2006_2009.pdf.

25. SUPERINTENDENCIA DE SOCIEDADES (Abril 6 de 2000). Circular Externa No. 005. Consolidación de Estados Financieros. Disponible en: http://www. supersociedades.gov.co/superintendencia/normatividad/circulares-externas/Normatividad\%20Circulares\%20Externas/572.pdf.

26. SUPERINTENDENCIA DE SOCIEDADES (26 de noviembre de 1997). Circular Externa №. 030. Criterios generales para la aplicación del régimen legal de las matrices, subordinadas, situaciones de control y grupos empresariales y Estados Financieros Consolidados. Disponible en: http://www. supersociedades.gov.co/superintendencia/normatividad/circulares-externas/Normatividad\%20Circulares\%20Externas/557.pdf.

27. TIPOS DE INVESTIGACIÓN Y DISEÑO DE INVESTIGACIÓN (2010). Disponible en: http://metodologia02.blogspot.com/p/operacionalizacion-devariables.html.

28. TIPOS DE INVESTIGACIÓN (2008). Disponible en: http://www.rena.edu.ve/cuartaEtapa/metodologia/Tema4.html. 